\title{
Comparison of methanogen diversity of yak (Bos grunniens) and cattle (Bos taurus) from the Qinghai-Tibetan plateau, China
}

\author{
Xiao Dan Huang ${ }^{1}$, Hui Yin Tan², Ruijun Long ${ }^{1 *}$, Juan Boo Liang ${ }^{3}$ and André-Denis G Wright ${ }^{4}$
}

\begin{abstract}
Background: Methane emissions by methanogen from livestock ruminants have significantly contributed to the agricultural greenhouse gas effect. It is worthwhile to compare methanogen from "energy-saving" animal (yak) and normal animal (cattle) in order to investigate the link between methanogen structure and low methane production.

Results: Diversity of methanogens from the yak and cattle rumen was investigated by analysis of 16S rRNA gene sequences from rumen digesta samples from four yaks (209 clones) and four cattle (205 clones) from the Qinghai-Tibetan Plateau area (QTP). Overall, a total of 414 clones (i.e. sequences) were examined and assigned to 95 operational taxonomic units (OTUs) using MOTHUR, based upon a 98\% species-level identity criterion. Forty-six OTUs were unique to the yak clone library and 34 OTUs were unique to the cattle clone library, while 15 OTUs were found in both libraries. Of the 95 OTUs, 93 putative new species were identified. Sequences belonging to the Thermoplasmatales-affiliated Linage C (TALC) were found to dominate in both libraries, accounting for $80.9 \%$ and $62.9 \%$ of the sequences from the yak and cattle clone libraries, respectively. Sequences belonging to the Methanobacteriales represented the second largest clade in both libraries. However, Methanobrevibacter wolinii (QTPC 110) was only found in the cattle library. The number of clones from the order Methanomicrobiales was greater in cattle than in the yak clone library. Although the Shannon index value indicated similar diversity between the two libraries, the Libshuff analysis indicated that the methanogen community structure of the yak was significantly different than those from cattle.

Conclusion: This study revealed for the first time the molecular diversity of methanogen community in yaks and cattle in Qinghai-Tibetan Plateau area in China. From the analysis, we conclude that yaks have a unique rumen microbial ecosystem that is significantly different from that of cattle, this may also help to explain why yak produce less methane than cattle.
\end{abstract}

\section{Background}

Yak (Bos grunniens) and cattle (Bos taurus) separated about 4.4 to 5.3 million years ago [1]. While cattle have a worldwide distribution in most of the low lands, the yak has dominated in high lands especially around the Hindu Kush-Himalayan region and the Qinghai-Tibetan Plateau (QTP), ranging from 3,000 to $5,500 \mathrm{~m}$ above sea level. The yak is one of the world's most remarkable domestic animals, and has been reported as a typical four season grazing ruminant in the QTP [2]. In order to

\footnotetext{
* Correspondence: longrj@|zu.edu.cn

'International Centre for Tibetan Plateau Ecosystem Management, Lanzhou University, Lanzhou 730000, China

Full list of author information is available at the end of the article
}

adapt to the harsh environment with severe cold, less oxygen, strong ultra-violet (UV) radiation, and poor forage resources, yaks have evolved special adaptations in physiology, nutrient metabolism and foraging [3-8].

Recently, Shao et al [5] anatomically compared the yak tongue with the cattle tongue, and found that the yak tongue was better adapted to the harsh characteristics of Tibetan pasture. Other recent studies have shown that yaks have an efficient nitrogen metabolism, suggesting an adaptation mechanism to their low-N dietary ingestion under harsh grasslands conditions of the QTP area [8]. Subsequently, using the sulfur-hexafluoride (SF6) tracer technique, Ding et al [9] measured the enteric methane emissions of yak in the QTP area and 
showed that yaks produce less methane (per unit of live weight) compared to other ruminants, such as cattle.

Greenhouse gases have become a major issue in the world and ruminant livestock are an important source of global enteric methane. Enteric methane gas is produced by microorganisms, called methanogens, in the digestive tract of ruminant livestock during digestion of feed and represents a direct loss of gross energy intake that could more efficiently be used by the animal for increased productivity [10]. Thus, reducing enteric methane production could benefit ruminants energetically, provided that the digestion efficiency and animal production traits are not compromised. The yak has great potential as an "energy-saving" animal as many researchers around the world aim to find "low carbon" livestock.

The identification of inhibitors of methanogenesis is currently being explored. However, the successful use of these agents is dependant upon having a better understanding of the hydrogenotrophic microbial community in the rumen, which must be promoted in the absence of the methanogenic archaea for production benefits to occur. As a potential "low carbon" animal, yaks are adapted to a cold and high altitude environment and are reported to produce less methane than cattle per unit body weight [9]. Thus, the yak, which is well adapted to its environment, may harbor a rumen methanogen population that produces less methane than cattle. Therefore, it is necessary to study the hydrogenotrophic microbial community by comparing the rumen methanogen diversity of yaks and cattle. The phylogenetic analysis of bacterial diversity in yak has been studied previously $[11,12]$, whereas the methanogen diversity in yak has yet to be investigated.

This study aims to generate new knowledge pertaining to the rumen methanogens of the yak and will contribute to the identification of the microbiology that constitutes a low-methane emitting ruminant animal. To our knowledge, this is the first investigation on the diversity of rumen methanogens from the yak.

\section{Results}

\section{Sequence similarity analysis}

In the yak 16S rRNA gene clone library, a total of 227 clones were examined and 18 clones were identified as chimeras and excluded from further analyses. The remaining 209 clones revealed 134 unique sequences (Table 1). Of these, 109 sequences belonged to the Thermoplasmatales-affiliated Lineage C (TALC), with only $85.5 \%$ to $89.2 \%$ identity to Methanomassiliicoccus luminyensis. The remaining 25 sequences were related to archaeal taxa from the orders Methanobacteriales, Methanomicrobiales and Methanosarcinales. Of these 25 sequences, 20 belonged within the order Methanobacteriales and were broken down as follows: 12 sequences were
97.0\% to $98.3 \%$ related to Methanobrevibacter millerae, four sequences had $96.7 \%$ to $98.9 \%$ identity to Methanobrevibacter ruminantium, and four sequences were $96.2 \%$ to $97.5 \%$ related to Methanobrevibacter smithii. Only one sequence was related to methanogens from the order Methanomicrobiales, with $99.8 \%$ identity to Methanomicrobium mobile, whereas four sequences belonged to the order Methanosarcinales with only $91.7 \%$ to $92.9 \%$ identity to Methanimicrococcus blatticola.

In the cattle $16 \mathrm{~S}$ rRNA gene library, a total of 216 clones was examined, of which 11 clones were identified as chimeras and excluded from the analysis. The remaining 205 sequences revealed 113 unique sequences (Table 1). A total of 72 sequences (129 clones) were only $83.8 \%$ to $89.2 \%$ related to Methanomassiliicoccus luminyensis, whereas 33 sequences (44 clones) were $95.5 \%$ to 99.1\% related to methanogens belonging to the order Methanobacteriales and six sequences (20 clones) were 99.4 to $99.8 \%$ related to those belonging to the order Methanomicrobiales. The remaining two sequences (12 clones) were $92.5 \%$ and $92.8 \%$ related to Methanimicrococcus blatticola within the order Methanosarcinales. Within the Methanobacteriales, 27 of the 33 sequences were $96.0 \%$ to $99.1 \%$ identical to Methanobrevibacter millerae, two sequences (QTPC 9 and QTPC 15) were 97.6 to $98.4 \%$ related to Methanobrevibacter gottschalkii; one sequence (QTPC 70) was only $95.5 \%$ related to Methanobrevibacter arboriphilus; and three sequences (QTPC 112, QTPC 27 and QTPC 110) were 99\%. 96.8\% and $95.7 \%$ related to Methanobrevibacter ruminantium, Methanobrevibacter smithii and Methanobrevibacter wolinii, respectively.

Using a species-level identity criterion of $98 \%$ [13], 93 of the 95 OTUs had less than $98 \%$ identity to any valid recognized taxa, and may represent potential new methanogen species and strains.

\section{Statistical analysis of libraries}

The yak library had a Shannon index of $3.33 \pm 0.18$ while the cattle library had a Shannon index of $3.02 \pm 0.19$. Libshuff analysis showed that the differences between the yak and cattle libraries at $98 \%$ identity were significant $(P<0.0001)$.

\section{Phylogenetic placement of sequences}

Distance-matrix phylogenetic trees are provided showing the phylogenetic placement of the methanogen sequences from the yak and cattle (Figure 1) clone libraries. Methanogen sequences from yak and cattle grouped with methanogens from the uncharacterized TALC group (Figure 1b), as well as the orders Methanobacteriales, Methanomicrobiales, Methanosarcinales (Figure 1a).

In total, 414 clones were analyzed, revealing 247 unique sequences (134 sequences from yak and 113 
Table 1 Similarity values of rumen methanogens from yak and cattle from Qinghai-Tibetan Plateau, China

\begin{tabular}{|c|c|c|c|c|c|c|c|c|c|}
\hline \multicolumn{5}{|c|}{ Yak } & \multicolumn{5}{|c|}{ Cattle } \\
\hline 16S Sequence & Clones $^{\mathrm{a}}$ & OTU\# & Nearest Taxon & \% Seq ID & 16S Sequence & Clones $^{a}$ & OTU\# & Nearest Taxon & $\%$ Seq ID \\
\hline QTPYAK1 & 5 & 74 & Mms. luminyensis & 88.2 & QTPC1 & 2 & 82 & Mbb. millerae & 98.6 \\
\hline QTPYAK2 & 1 & 74 & Mms. luminyensis & 88.1 & QTPC2 & 1 & 82 & Mbb. millerae & 99.0 \\
\hline QTPYAK3 & 1 & 82 & Mbb. millerae & 97.9 & QTPC3 & 3 & 82 & Mbb. millerae & 98.2 \\
\hline QTPYAK4 & 1 & 82 & Mbb. millerae & 98.1 & QTPC4 & 4 & 49 & Mms. luminyensis & 87.9 \\
\hline QTPYAK5 & 1 & 82 & Mbb. millerae & 97.6 & QTPC5 & 1 & 63 & Mms. luminyensis & 88.4 \\
\hline QTPYAK6 & 1 & 82 & Mbb. millerae & 98.3 & QTPC6 & 4 & 49 & Mms. luminyensis & 87.8 \\
\hline QTPYAK7 & 2 & 82 & Mbb. millerae & 98.1 & QTPC7 & 1 & 33 & Mms. luminyensis & 87.8 \\
\hline QTPYAK8 & 1 & 84 & Mbb. millerae & 97.1 & QTPC8 & 1 & 82 & Mbb. millerae & 99.1 \\
\hline QTPYAK9 & 1 & 82 & Mbb. millerae & 98.0 & QTPC9 & 2 & 82 & Mbb. gottschalkii & 97.6 \\
\hline QTPYAK10 & 1 & 82 & Mbb. millerae & 98.2 & QTPC10 & 1 & 82 & Mbb. millerae & 98.3 \\
\hline QTPYAK11 & 1 & 83 & Mbb. millerae & 97.7 & QTPC11 & 1 & 82 & Mbb. millerae & 98.3 \\
\hline QTPYAK12 & 1 & 89 & Mbb. smithii & 96.3 & QTPC12 & 1 & 82 & Mbb. millerae & 97.7 \\
\hline QTPYAK13 & 1 & 50 & Mms. luminyensis & 87.9 & QTPC13 & 1 & 82 & Mbb. millerae & 98.4 \\
\hline QTPYAK14 & 2 & 51 & Mms. luminyensis & 88.8 & QTPC14 & 1 & 82 & Mbb. millerae & 98.7 \\
\hline QTPYAK15 & 2 & 36 & Mms. luminyensis & 87.1 & QTPC15 & 1 & 82 & Mbb. gottschalkii & 98.4 \\
\hline QTPYAK16 & 1 & 52 & Mms. luminyensis & 87.8 & QTPC16 & 1 & 10 & Mms. luminyensis & 87.1 \\
\hline QTPYAK17 & 3 & 49 & Mms. luminyensis & 88.2 & QTPC17 & 3 & 82 & Mbb. millerae & 98.0 \\
\hline QTPYAK18 & 1 & 53 & Mms. luminyensis & 88.0 & QTPC18 & 1 & 82 & Mbb. millerae & 97.9 \\
\hline QTPYAK19 & 1 & 16 & Mms. luminyensis & 87.0 & QTPC19 & 2 & 82 & Mbb. millerae & 97.9 \\
\hline QTPYAK20 & 1 & 68 & Mms. luminyensis & 87.4 & QTPC20 & 1 & 82 & Mbb. millerae & 98.3 \\
\hline QTPYAK21 & 1 & 4 & Mms. luminyensis & 88.0 & QTPC21 & 1 & 82 & Mbb. millerae & 98.5 \\
\hline QTPYAK22 & 2 & 49 & Mms. luminyensis & 88.1 & QTPC22 & 1 & 82 & Mbb. millerae & 98.4 \\
\hline QTPYAK23 & 2 & 49 & Mms. luminyensis & 88.1 & QTPC23 & 2 & 82 & Mbb. millerae & 97.7 \\
\hline QTPYAK24 & 2 & 61 & Mms. luminyensis & 88.4 & QTPC24 & 1 & 82 & Mbb. millerae & 98.3 \\
\hline QTPYAK25 & 1 & 62 & Mms. luminyensis & 88.6 & QTPC25 & 2 & 82 & Mbb. millerae & 98.1 \\
\hline QTPYAK26 & 4 & 49 & Mms. luminyensis & 88.0 & QTPC26 & 2 & 82 & Mbb. millerae & 97.9 \\
\hline QTPYAK27 & 1 & 49 & Mms. luminyensis & 87.8 & QTPC27 & 1 & 86 & Mbb. smithii & 96.8 \\
\hline QTPYAK28 & 1 & 49 & Mms. luminyensis & 88.5 & QTPC28 & 1 & 49 & Mms. luminyensis & 87.9 \\
\hline QTPYAK29 & 1 & 49 & Mms. luminyensis & 87.8 & QTPC29 & 2 & 28 & Mms. luminyensis & 86.8 \\
\hline QTPYAK30 & 2 & 85 & Mbb. smithii & 97.5 & QTPC30 & 6 & 80 & Mmb. mobile & 99.7 \\
\hline QTPYAK31 & 2 & 82 & Mbb. millerae & 98.3 & QTPC31 & 1 & 80 & Mmb. mobile & 99.7 \\
\hline QTPYAK32 & 3 & 88 & Mbb. millerae & 97.0 & QTPC32 & 1 & 80 & Mmb. mobile & 99.4 \\
\hline QTPYAK33 & 1 & 90 & Mbb. millerae & 97.0 & QTPC33 & 3 & 80 & Mmb. mobile & 99.5 \\
\hline QTPYAK34 & 1 & 70 & Mms. luminyensis & 88.5 & QTPC34 & 2 & 80 & Mmb. mobile & 99.5 \\
\hline QTPYAK35 & 1 & 70 & Mms. luminyensis & 88.4 & QTPC35 & 7 & 80 & Mmb. mobile & 99.8 \\
\hline QTPYAK36 & 1 & 70 & Mms. luminyensis & 88.4 & QTPC36 & 4 & 70 & Mms. luminyensis & 88.0 \\
\hline QTPYAK37 & 1 & 70 & Mms. luminyensis & 88.3 & QTPC37 & 3 & 16 & Mms. luminyensis & 86.6 \\
\hline QTPYAK38 & 1 & 77 & Mms. luminyensis & 87.9 & QTPC38 & 5 & 39 & Mms. luminyensis & 86.6 \\
\hline QTPYAK39 & 3 & 70 & Mms. luminyensis & 88.5 & QTPC39 & 9 & 39 & Mms. luminyensis & 86.5 \\
\hline QTPYAK40 & 1 & 70 & Mms. luminyensis & 88.4 & QTPC40 & 2 & 39 & Mms. luminyensis & 86.7 \\
\hline QTPYAK41 & 1 & 70 & Mms. luminyensis & 88.4 & QTPC41 & 1 & 16 & Mms. luminyensis & 86.5 \\
\hline QTPYAK42 & 1 & 70 & Mms. luminyensis & 88.6 & QTPC42 & 3 & 58 & Mms. luminyensis & 87.8 \\
\hline QTPYAK43 & 4 & 74 & Mms. luminyensis & 87.8 & QTPC43 & 2 & 16 & Mms. luminyensis & 86.7 \\
\hline QTPYAK44 & 4 & 74 & Mms. luminyensis & 87.9 & QTPC44 & 3 & 58 & Mms. luminyensis & 88.3 \\
\hline
\end{tabular}


Table 1 Similarity values of rumen methanogens from yak and cattle from Qinghai-Tibetan Plateau, China (Continued)

\begin{tabular}{|c|c|c|c|c|c|c|c|c|}
\hline QTPYAK45 & 2 & 74 & Mms. luminyensis & 87.9 & QTPC45 & 4 & 69 & Mms. luminyensis \\
\hline QTPYAK46 & 1 & 71 & Mms. luminyensis & 88.6 & QTPC46 & 1 & 56 & Mms. luminyensis \\
\hline QTPYAK47 & 7 & 81 & Mmc. blatticola & 92.9 & QTPC47 & 1 & 55 & Mms. luminyensis \\
\hline QTPYAK48 & 2 & 81 & Mmc. blatticola & 92.9 & QTPC48 & 1 & 67 & Mms. luminyensis \\
\hline QTPYAK49 & 1 & 81 & Mmc. blatticola & 91.7 & QTPC49 & 1 & 82 & Mbb. millerae \\
\hline QTPYAK50 & 2 & 81 & Mmc. blatticola & 92.6 & QTPC50 & 1 & 1 & Mms. luminyensis \\
\hline QTPYAK51 & 2 & 49 & Mms. luminyensis & 88.7 & QTPC51 & 1 & 82 & Mbb. millerae \\
\hline QTPYAK52 & 1 & 37 & Mms. luminyensis & 88.0 & QTPC52 & 1 & 82 & Mbb. millerae \\
\hline QTPYAK53 & 1 & 57 & Mms. luminyensis & 87.7 & QTPC53 & 1 & 82 & Mbb. millerae \\
\hline QTPYAK54 & 2 & 74 & Mms. luminyensis & 87.9 & QTPC55 & 1 & 82 & Mbb. millerae \\
\hline QTPYAK55 & 1 & 76 & Mms. luminyensis & 87.1 & QTPC56 & 1 & 25 & Mms. luminyensis \\
\hline QTPYAK56 & 2 & 72 & Mms. luminyensis & 87.5 & QTPC57 & 1 & 41 & Mms. luminyensis \\
\hline QTPYAK57 & 1 & 72 & Mms. luminyensis & 87.6 & QTPC58 & 2 & 94 & Mbb. millerae \\
\hline QTPYAK58 & 2 & 72 & Mms. luminyensis & 87.9 & QTPC59 & 2 & 55 & Mms. luminyensis \\
\hline QTPYAK59 & 1 & 75 & Mms. luminyensis & 87.3 & QTPC60 & 4 & 55 & Mms. luminyensis \\
\hline QTPYAK60 & 1 & 70 & Mms. luminyensis & 88.1 & QTPC61 & 2 & 55 & Mms. luminyensis \\
\hline QTPYAK61 & 1 & 39 & Mms. luminyensis & 86.3 & QTPC62 & 1 & 73 & Mms. luminyensis \\
\hline QTPYAK62 & 2 & 39 & Mms. luminyensis & 86.2 & QTPC63 & 1 & 41 & Mms. luminyensis \\
\hline QTPYAK63 & 2 & 39 & Mms. luminyensis & 86.5 & QTPC64 & 1 & 91 & Mbb. millerae \\
\hline QTPYAK64 & 4 & 46 & Mms. luminyensis & 86.7 & QTPC65 & 1 & 73 & Mms. luminyensis \\
\hline QTPYAK65 & 1 & 49 & Mms. luminyensis & 88.4 & QTPC66 & 1 & 40 & Mms. luminyensis \\
\hline QTPYAK67 & 2 & 80 & Mmb. mobile & 99.8 & QTPC68 & 1 & 7 & Mms. luminyensis \\
\hline QTPYAK68 & 1 & 64 & Mms. luminyensis & 87.5 & QTPC69 & 1 & 82 & Mbb. millerae \\
\hline QTPYAK69 & 2 & 93 & Mbb. ruminantium & 96.7 & QTPC70 & 1 & 94 & Mbb. arboriphilus \\
\hline QTPYAK70 & 1 & 87 & Mbb. ruminantium & 96.8 & QTPC71 & 1 & 59 & Mms. luminyensis \\
\hline QTPYAK71 & 1 & 87 & Mbb. smithii & 96.5 & QTPC72 & 1 & 59 & Mms. luminyensis \\
\hline QTPYAK72 & 1 & 32 & Mms. luminyensis & 86.8 & QTPC73 & 3 & 1 & Mms. luminyensis \\
\hline QTPYAK73 & 1 & 92 & Mbb. ruminantium & 98.1 & QTPC74 & 10 & 16 & Mms. luminyensis \\
\hline QTPYAK74 & 1 & 92 & Mbb. ruminantium & 98.9 & QTPC75 & 1 & 16 & Mms. luminyensis \\
\hline QTPYAK75 & 1 & 35 & Mms. luminyensis & 87.2 & QTPC76 & 2 & 16 & Mms. luminyensis \\
\hline QTPYAK76 & 1 & 49 & Mms. luminyensis & 88.4 & QTPC77 & 6 & 16 & Mms. luminyensis \\
\hline QTPYAK77 & 1 & 42 & Mms. luminyensis & 88.3 & QTPC78 & 1 & 16 & Mms. luminyensis \\
\hline QTPYAK78 & 1 & 42 & Mms. luminyensis & 87.5 & QTPC79 & 1 & 24 & Mms. luminyensis \\
\hline QTPYAK79 & 1 & 16 & Mms. luminyensis & 86.6 & QTPC80 & 1 & 16 & Mms. luminyensis \\
\hline QTPYAK80 & 1 & 16 & Mms. luminyensis & 86.7 & QTPC81 & 1 & 16 & Mms. luminyensis \\
\hline QTPYAK81 & 10 & 16 & Mms. luminyensis & 86.6 & QTPC82 & 1 & 20 & Mms. luminyensis \\
\hline QTPYAK82 & 1 & 16 & Mms. luminyensis & 86.5 & QTPC83 & 1 & 9 & Mms. luminyensis \\
\hline QTPYAK83 & 1 & 16 & Mms. luminyensis & 86.4 & QTPC84 & 1 & 24 & Mms. luminyensis \\
\hline QTPYAK84 & 3 & 16 & Mms. luminyensis & 86.4 & QTPC85 & 1 & 26 & Mms. luminyensis \\
\hline QTPYAK85 & 1 & 16 & Mms. luminyensis & 86.4 & QTPC86 & 2 & 48 & Mms. luminyensis \\
\hline QTPYAK86 & 1 & 16 & Mms. luminyensis & 86.7 & QTPC87 & 1 & 21 & Mms. luminyensis \\
\hline QTPYAK87 & 1 & 16 & Mms. luminyensis & 86.7 & QTPC88 & 1 & 23 & Mms. luminyensis \\
\hline QTPYAK88 & 1 & 16 & Mms. luminyensis & 87.0 & QTPC89 & 1 & 22 & Mms. luminyensis \\
\hline QTPYAK89 & 1 & 16 & Mms. luminyensis & 86.6 & QTPC90 & 1 & 39 & Mms. luminyensis \\
\hline QTPYAK90 & 1 & 16 & Mms. luminyensis & 86.7 & QTPC91 & 1 & 42 & Mms. luminyensis \\
\hline QTPYAK91 & 2 & 16 & Mms. luminyensis & 86.5 & QTPC92 & 1 & 2 & Mms. luminyensis \\
\hline
\end{tabular}


Table 1 Similarity values of rumen methanogens from yak and cattle from Qinghai-Tibetan Plateau, China (Continued)

\begin{tabular}{|c|c|c|c|c|c|c|c|c|c|}
\hline QTPYAK92 & 1 & 16 & Mms. luminyensis & 87.4 & QTPC93 & 1 & 2 & Mms. luminyensis & 88.0 \\
\hline QTPYAK93 & 1 & 16 & Mms. luminyensis & 87.2 & QTPC94 & 1 & 1 & Mms. luminyensis & 87.7 \\
\hline QTPYAK94 & 6 & 16 & Mms. luminyensis & 86.5 & QTPC95 & 6 & 81 & Mmc. blatticola & 92.8 \\
\hline QTPYAK95 & 2 & 16 & Mms. luminyensis & 86.3 & QTPC96 & 6 & 81 & Mmc. blatticola & 92.5 \\
\hline QTPYAK96 & 2 & 16 & Mms. luminyensis & 87.2 & QTPC97 & 2 & 39 & Mms. luminyensis & 87.1 \\
\hline QTPYAK97 & 1 & 16 & Mms. luminyensis & 86.3 & QTPC98 & 1 & 39 & Mms. Iuminyensis & 87.2 \\
\hline QTPYAK98 & 1 & 15 & Mms. luminyensis & 87.2 & QTPC99 & 1 & 47 & Mms. luminyensis & 86.4 \\
\hline QTPYAK99 & 1 & 27 & Mms. luminyensis & 87.1 & QTPC100 & 1 & 59 & Mms. luminyensis & 88.5 \\
\hline QTPYAK100 & 1 & 27 & Mms. luminyensis & 87.4 & QTPC101 & 1 & 79 & Mms. luminyensis & 87.1 \\
\hline QTPYAK101 & 1 & 14 & Mms. luminyensis & 87.0 & QTPC102 & 1 & 5 & Mms. luminyensis & 88.4 \\
\hline QTPYAK102 & 1 & 24 & Mms. luminyensis & 86.7 & QTPC103 & 1 & 6 & Mms. luminyensis & 87.6 \\
\hline QTPYAK103 & 1 & 12 & Mms. luminyensis & 87.3 & QTPC104 & 1 & 66 & Mms. luminyensis & 88.5 \\
\hline QTPYAK104 & 1 & 19 & Mms. luminyensis & 85.5 & QTPC105 & 1 & 29 & Mms. luminyensis & 86.4 \\
\hline QTPYAK105 & 1 & 13 & Mms. luminyensis & 87.5 & QTPC106 & 1 & 45 & Mms. luminyensis & 87.4 \\
\hline QTPYAK106 & 1 & 17 & Mms. luminyensis & 85.9 & QTPC107 & 1 & 54 & Mms. luminyensis & 87.7 \\
\hline QTPYAK107 & 1 & 17 & Mms. luminyensis & 86.4 & QTPC108 & 1 & 48 & Mms. luminyensis & 86.7 \\
\hline QTPYAK108 & 1 & 11 & Mms. luminyensis & 86.8 & QTPC109 & 1 & 30 & Mms. luminyensis & 86.5 \\
\hline QTPYAK109 & 3 & 16 & Mms. luminyensis & 86.5 & QTPC110 & 1 & 95 & Mbb. wolinii & 95.7 \\
\hline QTPYAK110 & 1 & 18 & Mms. luminyensis & 86.2 & QTPC111 & 1 & 39 & Mms. luminyensis & 86.3 \\
\hline QTPYAK111 & 1 & 16 & Mms. luminyensis & 86.8 & QTPC112 & 1 & 92 & Mbb. ruminantium & 99.0 \\
\hline QTPYAK112 & 2 & 16 & Mms. luminyensis & 85.9 & QTPC113 & 1 & 43 & Mms. luminyensis & 88.4 \\
\hline QTPYAK113 & 1 & 18 & Mms. luminyensis & 86.3 & QTPC114 & 1 & 42 & Mms. luminyensis & 87.7 \\
\hline QTPYAK114 & 2 & 16 & Mms. luminyensis & 86.2 & & & & & \\
\hline QTPYAK115 & 1 & 16 & Mms. luminyensis & 86.3 & & & & & \\
\hline QTPYAK116 & 1 & 34 & Mms. luminyensis & 87.2 & & & & & \\
\hline QTPYAK117 & 2 & 34 & Mms. luminyensis & 87.7 & & & & & \\
\hline QTPYAK118 & 1 & 8 & Mms. luminyensis & 88.1 & & & & & \\
\hline QTPYAK119 & 2 & 34 & Mms. luminyensis & 87.9 & & & & & \\
\hline QTPYAK120 & 1 & 41 & Mms. luminyensis & 86.3 & & & & & \\
\hline QTPYAK121 & 1 & 89 & Mbb. smithii & 96.2 & & & & & \\
\hline QTPYAK122 & 1 & 44 & Mms. luminyensis & 87.9 & & & & & \\
\hline QTPYAK123 & 1 & 58 & Mms. luminyensis & 87.9 & & & & & \\
\hline QTPYAK124 & 1 & 78 & Mms. luminyensis & 88.1 & & & & & \\
\hline QTPYAK125 & 1 & 59 & Mms. luminyensis & 89.1 & & & & & \\
\hline QTPYAK126 & 1 & 59 & Mms. luminyensis & 89.2 & & & & & \\
\hline QTPYAK127 & 1 & 74 & Mms. luminyensis & 88.1 & & & & & \\
\hline QTPYAK128 & 1 & 2 & Mms. luminyensis & 87.7 & & & & & \\
\hline QTPYAK129 & 2 & 38 & Mms. luminyensis & 88.2 & & & & & \\
\hline QTPYAK130 & 1 & 65 & Mms. luminyensis & 88.7 & & & & & \\
\hline QTPYAK132 & 1 & 58 & Mms. luminyensis & 88.9 & & & & & \\
\hline QTPYAK133 & 1 & 60 & Mms. luminyensis & 88.7 & & & & & \\
\hline QTPYAK134 & 1 & 2 & Mms. luminyensis & 87.3 & & & & & \\
\hline QTPYAK135 & 1 & 21 & Mms. luminyensis & 87.1 & & & & & \\
\hline
\end{tabular}



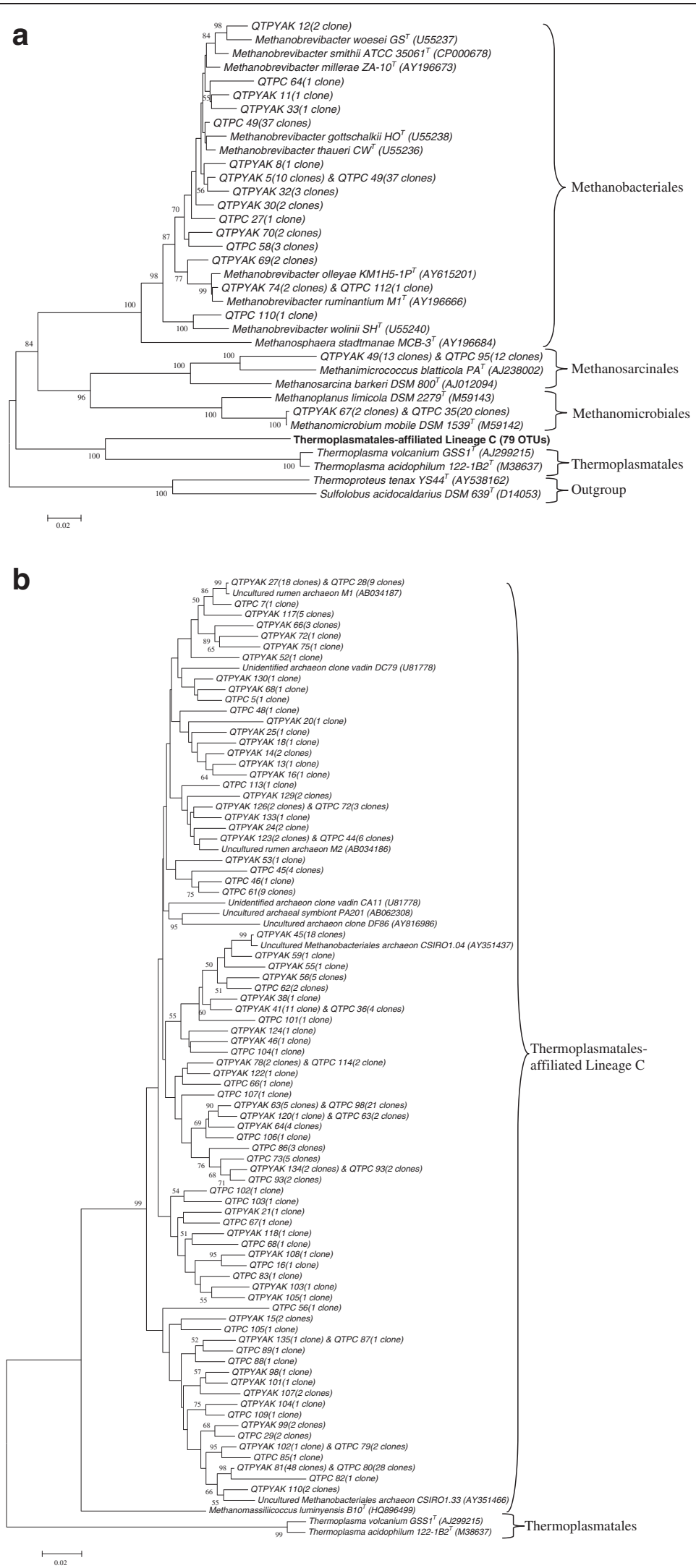

Figure 1 (See legend on next page.) 
(See figure on previous page.)

Figure 1 Phylogenetic analysis of methanogen partial 16S rRNA sequences from yak and cattle clone library inferred using MEGA (ver. 5). Of the 414 clones examined, 209 clones from yak and 205 clones from cattle were assigned to 95 OTUs by MOTHUR using a 98\% species level identity. These 95 OTUs are shown by representative sequences on the tree. In which, 16 OTUs from non-TALC group are presented in Figure 1a, and 79 OTUs from TALC group are presented in Figure 1b. GenBank accession number are indicated in parentheses and bootstrap values (>50\%) from 1000 replications are indicated on the tree.The scale bar corresponds to 2 changes per 100 positions.

sequences from cattle), which were assigned to 95 OTUs (79 TALC and 16 non-TALC). Examination of these 95 OTUs revealed that, 46 OTUs were unique to the yak clone library and 34 OTUs were unique to the cattle clone library (Figure 1a and 1b), while 15 OTUs (15.8\%) were found in both libraries as shared OTUs.

\section{Discussion}

The Yak is a key species in the Qinghai Tibetan Plateau. It provides herders with milk, meat, fiber, fuel and draught power, but also plays a key role in the management of the alpine rangeland ecosystem [14]. This ecological niche is unique and no other animal species can substitute the yak at such harsh environments (i.e. high altitude with lower oxygen levels and freezing temperatures in the winter). Research on the yak production system is therefore highly strategic and in recent years, adaptations of physiology, nitrogen and energy metabolism, histological variations, and foraging behavior to the harsh forage environment have been revealed [3-8]. However, research focusing on the rumen microbiota of the yak, has been limited until now. Based upon the Libshuff analysis, the current study has shown that the community structure of the methanogens resident in the yak is significantly different $(\mathrm{p}<0.0001)$ from that of cattle, with only 15 of the 95 OTUs shared between the two libraries.

The rumen is a unique environment which inhabits billions of microorganisms, including bacteria, methanogenic archaea, protozoa and fungi. Common species of methanogens isolated from rumen belong to the genera, Methanobrevibacter, Methanomicrobium, Methanobacterium and Methanosarcina $[15,16]$. In the present study, the majority of methanogen sequences were very distantly related to Methanomassiliicoccus luminyensis (Table 1) and were found to belong to the Thermoplasmatales-affiliated Lineage $C$, a group of uncultivated and uncharacterized rumen archaea that is a distantly related sister group to the order Thermoplasmatales (Figures 1). Tajima et al [17] also reported the methanogen diversity of the bovine rumen exhibited high degrees of similarity to uncultured archaea which were distantly related to the order Thermoplasmatales. Wright et al [18] also reported that 18 of 26 unique sequences from Australia sheep had 72 to $75 \%$ identity to Thermoplasmatales and were considered as predominant sequences in the rumen. In present study, within the TALC clade, few unique OTUs from yak and cattle libraries were highly related to the clones M1and M2 from Holstein cattle in Japan [17], clones CSIRO 1.04 and CSIRO 1.33 from sheep in Western Australia [18], and clones vadin CA11 and vadin DC79 from a wine anaerobic digester in France [19].

The distribution of 16S rRNA gene sequences within the orders of Methanobacteriales and Methanomicrobiales also varied between yak and cattle clone libraries. From the results, it was apparent that a greater percentage of the methanogen population from the orders of Methanobacteriales (21.5\% vs $12.4 \%$ ) and Methanomicrobiales $(9.8 \%$ vs $0.96 \%)$ were found in the rumen of cattle as compared to the yak.

Zhou et al [20] studied the methanogen diversity in cattle with different feed efficiencies and reported that differences at the strain and genotype levels of metagenomic ecology were found to be associated with feed efficiency in the host regardless of the population of methanogens. It was also suggested that the microbial ecology at the species, strain and genus levels in the rumen may play important roles in contributing to the difference in the methane gas production.

A recent investigation found that condensed tannins could exhibit a reduction in methane production in an in vitro gas production test [21]. Further investigation into the diversity of $16 \mathrm{~S}$ rRNA gene library of rumen methanogen in the condensed tannin treatment library revealed $21.9 \%$ higher diversity of sequences related to the TALC methanogens and a lower diversity of those associated with orders Methanobacteriales (15.1\%) and Methanomicrobiales (6.8\%) [22]. This shows a possible association between reduction in methane production and diversity of rumen methanogen. In the current study, yak has present higher methanogen diversity and significant different methanogen community structures compared with cattle (Figure 1). While there are many factors which may explain these differences in methanogen diversity, it is possible that these differences between the methanogen diversity in yak and cattle could be related to the significant difference in enteric methane production by both these ruminant species.

Long [23] reported a significantly high level of propionic acid, which leads to efficient energy utilization and this further suggested a low methane production in yak. Yak has also been found to exhibit lower methane output [9]. In the present study, yak had higher levels of acetate, proprionate, isobutyric, isovaleric and total volatile fatty acids 
than cattle, but cattle had higher acetate to proprionate $(\mathrm{A} / \mathrm{P})$ ratios (Table 2 ). This may also suggest different methanogenesis pathways. Therefore, the diversity and community structure of methanogens in yak, which is the lower methane producing ruminant species in current study, correlates with data reported by Tan et al [22].

Wright et al [24] revealed 65 sequences of methanogens by phylogenetic analysis from the Australian sheep rumen, and 62 of them belonged to the genus Methanobrevibacter. They were grouped with Methanobrevibacter NT7, Methanobrevibacter SM9, Methanobrevibacter M6, Methanobrevibacter ruminantium, Methanobrevibacter acididurans and Methanobrevibacter thaueri. From the present study (Figures 1a and 1b), clones related to Methanobrevibacter olleyae, Methanobrevibacter ruminantium, Methanobrevibacter woesei, Methanobrevibacter smithii, Methanobrevibacter millerae, Methanobrevibacter gottschalkii, and Methanobrevibacter thaueri were reported in the yak. However, in contrast with the yak library, Methanobrevibacter wolinii was only found in the cattle library. Clones related to Methanimicrococcus blatticola and Methanomicrobium mobile were found in both libraries.

Bacteria and methanogens has constantly interacted with each other in the rumen microbial communities [25], Sustainable growth of bacteria and methanogen in syntrophic communities depend on transfer of hydrogen and formate and reverse electron transfer [26]. In the present study, methanogens from the TALC cluster were the dominant sequences in the yak and cattle rumen in the QTP area. However, the metabolic mechanism of this methanogen group is not yet clear; the investigation of fermentive bacteria species in yak and cattle could help understanding these syntrophic microbial communities.

Table 2 The concentrations of volatile fatty acids from yak and cattle rumen samples

\begin{tabular}{|c|c|c|c|c|}
\hline $\begin{array}{l}\text { Volatile fatty } \\
\text { acids }\end{array}$ & $\begin{array}{c}\text { Yak } \\
(\mathrm{mmol} / \mathrm{L})\end{array}$ & $\begin{array}{c}\text { Cattle } \\
(\mathrm{mmol} / \mathrm{L})\end{array}$ & $\begin{array}{l}\text { Standard } \\
\text { error }\end{array}$ & Significance \\
\hline Acetate & 58.56 & 42.57 & 3.18 & $p<0.004$ \\
\hline Propionate & 12.13 & 7.35 & 0.93 & $p<0.001$ \\
\hline Isobutyric & 0.88 & 0.60 & 0.06 & $p<0.016$ \\
\hline Butyrate & 9.03 & 7.25 & 0.49 & $p<0.09$ \\
\hline Isovaleric & 1.02 & 0.51 & 0.12 & $p<0.027$ \\
\hline Valeric & 0.07 & 0.13 & 0.06 & $p<0.728$ \\
\hline $\begin{array}{l}\text { Total volatile } \\
\text { fatty acids }\end{array}$ & 81.69 & 58.41 & 4.61 & $p<0.001$ \\
\hline $\begin{array}{c}\text { A/P (Acetate to } \\
\text { Propionate) }\end{array}$ & 4.83 & 5.80 & 0.19 & $p<0.004$ \\
\hline
\end{tabular}

* Concentrations of volatile fatty acids was analysed by gas chromatograph equipped with a DB-FFAP column (30 $\mathrm{m} \times 0.25 \mu \mathrm{m} \times 0.25 \mu \mathrm{m}$; Agilent Technologies).

\section{Conclusions}

The current study revealed for the first time the molecular diversity of methanogen community in yaks and cattle in Qinghai-Tibetan Plateau area in China. The differences in methanogen diversity found in the present study, may help to explain, to some extent, the differences associated with the low methane production contributed to the adaptation of the yak to the harsh forage environment in the Qinghai-Tibetan plateau. Yaks have co-evolved with a unique rumen microbial ecosystem that is significantly different from that of cattle, even when feed similar diets. Understanding these particularities will yield development of technology for reducing methane emission intensity by optimizing dietary conditions to exploit the full potential of the yak ruminal ecosystem and function. However, native grazing might be a limited factor for this experiment, since feed intake could significantly influence the rumen microbiota. This study also contributes to the understanding of the specific features of the rumen microbial ecosystem of yaks which have adapted to high altitude ecosystems which may help to explain the differential rates of methanogenesis compared to cattle.

\section{Methods}

\section{Animals and diet}

Samples of individual rumen contents were obtained from four domestic cattle (BW: $160 \pm 5 \mathrm{~kg}$, Age: $4 \pm$ 0.4 years) and four domesticated yaks (body weight: 180 $\pm 5 \mathrm{~kg}$, Age: $4 \pm 0.6$ years) in the Qinghai Tibetan Plateau (QTP) in China. The animals were maintained outdoors, grazing a Kobresia pasture. Approximately $100 \mathrm{ml}$ of rumen contents were collected using a $1.5 \mathrm{~cm}$ diameter stomach tube attached to an electric pump. The animal sampling procedure strictly followed the rules and regulations of experimental field management protocols (file No: 2010-1 and 2010-2) which were approved by the Lanzhou University. Rumen contents were filtered through four layers of sterilized gauze and the rumen digesta was immediately transferred into sterile bottles and stored in liquid nitrogen until needed for DNA extraction and volatile fatty acids concentration analysis (Table 2).

\section{DNA extraction and PCR}

Genomic DNA was extracted from $300 \mu$ aliquots of the eight ( 4 yak and 4 cattle) thawed rumen samples using the QIAamp ${ }^{\circledR}$ DNA Stool kit (QIAGEN, Germany). The DNA extraction procedure was carried out in triplicate.

The methanogen-specific primers, Met86F (5'- GCT CAG TAA CAC GTG G-3') [27] and Met1340R (5' - CGG TGT GTG CAA GGA G-3') [27] were used to PCR amplify the $16 \mathrm{~S}$ rRNA gene using the following thermal cycling conditions: initial denaturation of 5 min 
at $94^{\circ} \mathrm{C}, 40$ cycles of denaturation at $94^{\circ} \mathrm{C}$ for $30 \mathrm{~s}$, annealing at $58^{\circ} \mathrm{C}$ for $1 \mathrm{~min}$, extension at $72^{\circ} \mathrm{C}$ for $90 \mathrm{~s}$, and a final extension at $72^{\circ} \mathrm{C}$ for $10 \mathrm{~min}$. Each PCR mixture contained $1 \mu \mathrm{l}(20 \mathrm{ug})$ of genomic DNA, $200 \mathrm{nM}$ of each primer, $10 \mu \mathrm{M}$ of dNTP (i-DNA Biotechnology Pte Ltd, Singapore), $1 \mathrm{x}$ VioTaq ${ }^{\circledR}$ reaction buffer, $0.5 \mathrm{U}$ of VioTaq $^{\circledR}$ Taq DNA polymerase (Viogene, Taiwan) and deionized water, in a final volume of $20 \mu \mathrm{l}$. PCR product of about $1.3 \mathrm{~kb}$ was isolated from the agarose gel and purified using MEGAquick-spin ${ }^{\text {Tw }}$ PCR and an agarose gel DNA extraction Kit (iNtRON Biotechnology, Seongnam, South Korea).

\section{Cloning, sequencing, and analyses}

Using chemical transformation, purified PCR products were cloned into the pCR $2.1^{\circledR}$ TOPO vector using the PCR $2.1^{\circledR}$ TOPO TA Cloning Kit (Invitrogen Ltd, USA). Recombinant colonies were picked and plasmid DNA was extracted using DNA-spin ${ }^{\text {Tx }}$ Plasmid DNA Extraction Kit (iNtRON Biotechnology, Korea). Sequencing was performed with an automated sequencer ABI 3730 xl using Big Dye Chemistry.

All sequences were aligned with ClustalW [28] in BioEdit software, and the Basic Local Alignment Search Tool (BLAST) [29] was used to determine the identity to the nearest recognized species available in the GenBank database. A species-level cutoff of 98\% [13] was used to assign sequences to OTUs and chimeras were identified using the Mallard program [30].

MOTHUR ver. 1.23.1 [31] was used to assign sequences to OTUs, and within MOTHUR, the Shannon index [32] and Libshuff analysis were used to assess the methanogen diversity and community structure of each library, respectively.

\section{Phylogenetic analysis}

A total of 27 archaeon sequences from GenBank were used as reference sequences, and two members of the Crenarchaeota, Sulfolobus acidocaldarius (D14053) and Thermoproteus tenax (AY538162), were the outgroup. All 16S rRNA gene clone sequences and the reference sequences were globally aligned using CLUSTAL W [33]. Phylogenetic analysis was performed by using MEGA ver 5.0 [34] using the neighbor-joining algorithm [35], with 1,000 bootstrap resamplings of the dataset [36]. Evolutionary distances between pairs of nucleotide sequences were calculated using Kimura two-parameter model [37].

\section{Nucleotide accession numbers}

Nucleotide sequences were designed with the prefix QTPYAK (Qinghai-Tibetan Plateau Yak) to represent 16S rRNA gene sequences from the yak clone library, and QTPC (Qinghai-Tibetan Plateau Cattle) for those from the cattle clone library. Prefixes were followed by the identification number of the unique sequence. All nucleotide sequences reported in this paper have been deposited in the GenBank database under the accession numbers JF807063 to JF807176 (i.e. cattle clones), excluding JF807116 (identical to JF807120); and JF807177 to JF807311 (i.e. Yak clones), excluding JF807307 (identical to JF807305).

\section{Competing interests}

The authors declare that they have no competing interests.

\section{Authors' contributions}

$\mathrm{XDH}$ sampled rumen contents from animals, performed DNA extractions, PCR amplification of methanogen 16S rRNA genes, clone library construction, data analysis, and drafted the manuscript. HYT contributed to all of the lab works and drafted the manuscript. RL conceived the study, sampled rumen contents from animals and drafted the manuscript. JBL contributed to the design of the study and drafted the manuscript; ADW performed data analysis, and drafted the manuscript. All authors read and approved the final manuscript.

\section{Acknowledgements}

This study was supported by the National Natural Science Foundation of China (NSFC) (project No.: 31170378), and the Scholarship Award for Excellent Doctoral Student Granted by Lanzhou University.

\section{Author details}

${ }^{1}$ International Centre for Tibetan Plateau Ecosystem Management, Lanzhou University, Lanzhou 730000, China. ${ }^{2}$ Institute of Bioscience, Universiti Putra Malaysia, Serdang 43400 UPM, Malaysia. ${ }^{3}$ Institute of Tropical Agriculture, Universiti Putra Malaysia, Serdang 43400 UPM, Malaysia. ${ }^{4}$ Department of Animal Science, University of Vermont, 570 Main Street, Burlington, Vermont 05405, USA.

\section{Received: 16 April 2012 Accepted: 11 October 2012}

Published: 19 October 2012

\section{References}

1. Gu Z, Zhao X, Li N, Wu C: Complete sequence of the yak (Bos grunniens) mitochondrial genome and its evolutionary relationship with other ruminants. Mol Phylogene Evol 2007, 42:248-255.

2. Long R, Apori SO, Castro FB, Orskov ER: Feed value of native forages of the Tibetan Plateau of China. Anim Feed Sci Technol 1999, 80:101-113.

3. Ding $L$, Long $R$, Yang $Y, X u S$, Wang $C$ : Behavioural responses by yaks in different physiological states (lactating, dry or replacement heifers), when grazing natural pasture in the spring (dry and germinating) season on the Qinghai-Tibetan plateau. Appl Anim Behav Sci 2007, 108:239-250.

4. Ding L, Long R, Shang Z, Wang C, Yang Y, Xu S: Feeding behaviour of yaks on spring, transitional, summer and winter pasture in the alpine region of the Qinghai-Tibetan plateau. Appl Anim Behav Sci 2008, 111:373-390.

5. Shao B, Long R, Ding Y, Wang J, Ding L, Wang H: Morphological adaptations of yak (Bos grunniens) tongue to the foraging environment of the Qinghai-Tibetan Plateau. J Anim Sci 2010, 88:2594-2603.

6. Wang H, Long R, Zhou W, Li X, Zhou J, Guo X: A comparative study on urinary purine derivative excretion of yak (Bos grunniens), cattle (Bos taurus), and crossbred (Bos taurus $\times$ Bos grunniens) in the Qinghai-Tibetan plateau, China. J Anim Sci 2009, 87:2355-2362.

7. Wang H, Long R, Liang JB, Guo X, Ding L, Shang Z: Comparison of nitrogen metabolism in yak (Bos grunniens) and Indigenous cattle (Bos taurus) on the Qinghai-Tibetan plateau. Asian-Aust J Anim Sci 2011, 24(6):766-773.

8. Guo XS, Zhang Y, Zhou JW, Long RJ, Xin GS, Qi B, Ding LM, Wang HC: Nitrogen metabolism and recycling in yaks (Bos grunniens) offered a forage-concentrate diet differing in N concentration. Anim Prod Sci 2012, 52:287-296

9. Ding $X$, Long $R$, Kreuzer M, Mi J, Yang B: Methane emissions from yak (Bos grunniens) steers grazing or kept indoors and fed diets with varying 
forage:concentrate ratio during the cold season on the Qinghai-Tibetan Plateau. Anim Feed Sci Technol 2010, 162:91-98.

10. Johnson KA, Johnson DE: Methane emissions from cattle. J Anim Sci 1995, 73:2483-2492

11. An D, Dong X, Dong Z: Prokaryote diversity in the rumen of yak (Bos grunniens) and Jinnan cattle (Bos taurus) estimated by 165 rDNA homology analyses. Anaerobe 2005, 11:207-215.

12. Yang L, Chen J, Cheng X, Xi D, Yang S, Deng W, Mao H: Phylogenetic analysis of $16 \mathrm{~S}$ rRNA gene sequences reveals rumen bacterial diversity in Yaks (Bos grunniens). Mol Biol Rep 2010, 37:553-562.

13. Wright A-DG, Northwood KS, Obispo NE: Rumen-like methanogens identified from the crop of the folivorous South American bird, the hoatzin (Opisthocomus hoazin). ISME 2009, 3:1120-1126.

14. Long R, Ding L, Shang Z, Guo X: The yak grazing system on the Qinghai-Tibetan plateau and its status. Rangeland J 2008, 30:241-246.

15. Wolin MJ, Miller TL, Stewart CS: Microbe-microbe interactions. In P N Hobson and C S Stewart (ed), The rumen microbial ecosystem. 2nd edition. New York, NY: Blackie Academic and Professional; 1997:467-491.

16. Jarvis GN, Strompl C, Burgess DM, Skillman LC, Moore ER, Joblin KN: Isolation and identification of ruminal methanogens from grazing cattle. Curr Microbiol 2000, 40:327-332.

17. Tajima K, Nagamine T, Matsui H, Nakamura M, Rustam I, Aminov Rl: Phylogenetic analysis of archaeal 165 rRNA libraries from the rumen suggests the existence of a novel group of archaea not associated with known methanogens. FEMS Microbiol Lett 2001, 200:67-72.

18. Wright A-DG, Toovey AF, Pimm CL: Molecular identification of methanogenic archaea from sheep in Queensland, Australia reveal more uncultured novel archaea. Anaerobe 2006, 12:134-139.

19. Godon JJ, Zumstein E, Dabert P, Habouzit F, Moletta R: Molecular microbial diversity of an anaerobic digestor as determined by small-subunit rDNA sequence analysis. Appl Environ Microbiol 1997, 63:2802-2813.

20. Zhou M, Hernandez-Sanabria E, Guan LL: Assessment of the microbial ecology of ruminal methanogens in cattle with different feed efficiencies. Appl Environ Microbiol 2009, 75:6524-6533.

21. Tan HY, Sieo CC, Abdullah N, Liang JB, Huang XD, Ho YW: Effects of condensed tannins from Leucaena on methane production, rumen fermentation and populations of methanogens and protozoa in vitro. Anim Feed Sci Technol 2011, 169:185-193.

22. Tan HY, Sieo CC, Lee CM, Abdullah N, Liang JB, Ho YW: Diversity of bovine rumen methanogens In vitro in the presence of condensed tannins, as determined by sequence analysis of $16 \mathrm{~S}$ rRNA gene library. J Microbiol 2011, 49:492-498.

23. Long R: Yak nutrition- a scientific basis. In The yak. 2nd edition. Edited by Gerald WN, Han JL, Long R. Thailand: RAP Publication; 2003:389-409.

24. Wright A-DG, Williams AJ, Winder B, Christophersen CT, Rodgers SL, Smith $\mathrm{KD}$ : Molecular diversity of rumen methanogens from sheep in Western Australia. Appl Environ Microb 2004, 70:1263-1270.

25. Stams AJM: Metabolic interactions between anaerobic bacteria in methanogenic environments. Antonie Leeuwenhoek 1994, 66:271-294.

26. Stams AJM, Plugge CM: Electron transfer in syntrophic communities of anaerobic bacteria and archaea. Nat Rev Microbiol 2009, 8:568-577.

27. Wright A-DG, Pimm CL: Improved strategy for presumptive identification of methanogens using $16 \mathrm{~S}$ riboprinting. J Microbiol Methods 2003, 55:337-349.

28. Hall TA: BioEdit: a user-friendly biological sequence alignment editor and analysis program for Windows 95/98/NT. Nucleic Acids Symp Ser 1999, 41:95-98.

29. Altschul SF, Madden TL, Schaffer AA, Zhang J, Zhang Z, Miller W, Lipman DJ: Gapped BLAST and PSI-BLAST: A new generation of protein database search programs. Nucleic Acids Res 1997, 25:3389-3402.

30. Ashelford KE, Chuzhanova NA, Fry JC, Jones AJ, Weightman AJ: At least 1 in $2016 \mathrm{~S}$ rRNA sequence records currently held in public repositories is estimated to contain substantial anomalies. Appl Environ Microbiol 2005, 71:7724-7736

31. Schloss PD, Westcott SL, Ryabin T, Hall JR, Hartmann M, Hollister EB, Lesniewski RA, Oakley BB, Parks DH, Robinson CJ, Sahl JW, Stres B, Thallinger GG, VanHorn DJ, Weber CF: Introducing mothur: open-source, platform-independent, community-supported software for describing and comparing microbial communities. Appl Environ Microbiol 2009, 75(23):7537-7541.

32. Shannon CE, Weaver W: The mathematical theory of communication Urbana: University of Illinois Press; 1949
33. Thompson JD, Higgins DG, Gibson TJ, Gibson TJ: CLUSTAL W: improving the sensitivity of progressive multiple sequence alignment through sequence weighting, position-specific gap penalties and weight matrix choice. Nucleic Acids Res 1994, 22:4673-4680.

34. Tamura K, Peterson D, Peterson N, Stecher G, Nei M, Kumar S: MEGA5: Molecular Evolutionary Genetics Analysis using Maximum Likelihood, Evolutionary Distance, and Maximum Parsimony Methods. Mol Biol Evol 2011, 28:2731-2739.

35. Saitou N, Nei M: The neighbor-joining method: a new method for reconstructing phylogenetic trees. Mol Biol Evol 1987, 4(4):406-425.

36. Felsenstein J: Confidence limits on phylogenies: an approach using the bootstrap. Evolution 1985, 39(4):783-791.

37. Kimura M: A simple method for estimating evolutionary rates of base substitutions through comparative studies of nucleotide sequences. J Mol Evol 1980, 16(2):111-120.

doi:10.1186/1471-2180-12-237

Cite this article as: Huang et al:: Comparison of methanogen diversity of yak (Bos grunniens) and cattle (Bos taurus) from the Qinghai-Tibetan plateau, China. BMC Microbiology 2012 12:237.

\section{Submit your next manuscript to BioMed Central and take full advantage of:}

- Convenient online submission

- Thorough peer review

- No space constraints or color figure charges

- Immediate publication on acceptance

- Inclusion in PubMed, CAS, Scopus and Google Scholar

- Research which is freely available for redistribution
C Biomed Central 